\title{
The Stabilizing Effects Of Dibutyltin Distearate On Some Properties Of Poly(Glycerol-Phthalate) Based Alkyd Coating Exposed To Outdoor Weather.
}

\author{
M. Turoti \\ Department of Chemistry, Ahmadu Bello University, Zaria - Nigeria.
}

\begin{abstract}
The effects of weathering on poly(glycerol-phthalate) based alkyd coatings of the unstabilised and stabilised samples using varying amounts of dibutyltin distearate have been studied. The weathering and stabilization processes were monitored using infrared spectroscopy,gel formation, colour development, tensile strength as well as determination of time of embrittlement. The results showed that, relative to the control, there were, reduction in hydroxyl moiety formation, lack of discoloration, increased gel content, enhanced tensile strength and elongated life span of the stabilised alkyd coating,This indicated effective reduction in degradation reactions in the presence of the test tin ester The $10 \times 10^{-4}$ mole\% dibutyltin distearate was the most effective concentration that stabilized the alkyd coatings.
\end{abstract}

\section{INTRODUCTION}

Alkyd resins constitute one of the oldest and yet one of the most versatile resins employed in the paints industry ${ }^{1}$. They are polycondensates of polyols and dicarboxylic acids modified with monocarboxylic acid which is reacted with the extra (unpolymerised) hydroxyl groups of the polyols. The major drawback of alkyd coatings is poor outdoor durability. This has restricted its use mainly to indoor coatings if used alone. The major degradation reactions associated with alkyds during exposure to outdoor environment have been identified $^{2}$ as photodegradation due to the attack of the UVB component of the solar radiation and hydrolytic degradation due to acid rain. The level of such degradative reactions would be expected to be greater iin tropical weather due to harsh sunshine, heavy rainfalls, and extreme harmattan as well as micrcobial components of the atmosphere.
In our previous articles ${ }^{3,4}$ an alkyd, poly(pentaerythritol phthalate), $\left[\mathrm{OCOC}_{6} \mathrm{H}_{4} \mathrm{COOCH}_{2} \mathrm{C}\left(\mathrm{CH}_{2} \mathrm{OCOR}\right)_{2} \mathrm{CH}_{2}\right.$ $\mathrm{O})_{\mathrm{n}}$,called AFTALAT VAT 3324 in the resin industry, modified with tall oil containing about $10-15 \% \mathrm{w} / \mathrm{w}$ conjugated acid) was proved to be stabilized by some dibutyltin dicarboxylates under outdoor conditions. The present report is on a different alkyd, poly(glycerol phthalate) $\left[\mathrm{OCOC}_{6} \mathrm{H}_{4} \mathrm{COOCH}_{2} \mathrm{CH}(\mathrm{OCOR}) \mathrm{CH}_{2} \mathrm{O}\right]_{\mathrm{n}} \mathrm{c}$ alled Nycil 5960 STX, a styrene vinylated alkyd resin based on soyabean and dehydrated castor oil (DCO) containing $40-42 \%$ conjugated acid under the similar exposure. No two alkyds have the same properties in the coating industry ${ }^{5}$. Therefore individual study of the degradation-

stabilization characteristics of alkyds, using any promising stabilizer under similar or different exposure conditions, is of paramount importance. 
Although diorganotin compounds such as dibutyltin maleate and dioctyltin thioglycollate have been used extensively as stabilizers in polymers particularly PVC, their use as stabilizers for an alkyd exposed to a tropical weather has just been recently reported $^{3,4}$. The present work is to show the effectiveness of the compounds as a stabilizer for another alkyd system. A comparison will also be made between some of the properties of the two alkyd alkyds thereby justifying the need for the pesent work.

\section{Materials}

\section{EXPERIMENTAL}

AR 5960 STX resin, a poly(glycerol-phthalate)(PGP) based alkyd, was obtained from Nycil Ltd.Lagos. Gasoline used as the thinner was obtained from the petrol station, but dried over anhydrous calcium chloride for $48 \mathrm{~h}$ and filtered before use.

\section{Substrates}

Glass sheet substrates were cut in dimensions of $10 \mathrm{~cm} \times 5 \mathrm{~cm} \times 0.2 \mathrm{~cm}$ cleansed by washing with soap solution and rinsed with distilled water before leaving in a desiccators to dry.

\section{Paper card film frames}

Cards of paper $5 \mathrm{~cm} \times 3 \mathrm{~cm}$ dimension were cut out of cardboard sheets. With a clean razor blade round holes of $2 \mathrm{~cm}$ diameter were cut on the paper cards for mounting films of test samples.

The infrared

spectrophotometer used was an ATIMATTSON USA GENESIS FTIR.

Synthesis and characterization of dibutyltin distearate

$30.30 \mathrm{~g}(0.1 \mathrm{~mole})$ of dibutyltin dichloride $\left(\mathrm{Bu}_{2} \mathrm{SnCl}_{2}\right)$ was dissolved in $200 \mathrm{~cm}^{3}$ of diethylether in a $500 \mathrm{~cm}^{3}$ separatory funnel. $11.20 \mathrm{~g}(0.2 \mathrm{~mole})$ of potassium hydroxide pellets was dissolved in $50 \mathrm{~cm}^{3}$ distilled water and the solution added to the ethereal solution of $\mathrm{Bu}_{2} \mathrm{SnCl}_{2}$ at room temperature. At about 30 minutes intervals the contents of the funnel were shaken for a period of $3 \mathrm{~h}$ and thereafter left to stand for another $3 \mathrm{hr}$. The white precipitate formed was filtered and purified as follows: unreacted $\mathrm{Bu}_{2} \mathrm{SnCl}_{2}$ was washed with ether until the filtrate did not produce any precipate with $\mathrm{KOH}$ solution. The reaction is

$\mathrm{Bu}_{2} \mathrm{SnCl}_{2}+2 \mathrm{KOH}$ room ter(ubdrat)

$$
\mathrm{Bu}_{2} \mathrm{Sn}(\mathrm{OH})_{2}+2 \mathrm{KCl}
$$

Any unreacted $\mathrm{KOH}$ was washed with distilled water until the filtrate did not show white precipitate after repeated tests with $\mathrm{AgNO}_{3}$ solution.

The white precipitate $\left(\mathrm{Bu}_{2} \mathrm{Sn}(\mathrm{OH})_{2}\right)$ was dehydrated by drying at $100^{\circ} \mathrm{C}$ to give dibutyltin oxide $\left(\mathrm{Bu}_{2} \mathrm{SnO}\right)$.

$$
\mathrm{Bu}_{2} \mathrm{Sn}(\mathrm{OH})_{2} \underset{100^{\circ} \mathrm{C}}{\stackrel{-\mathrm{H}_{2} \mathrm{O}}{\longrightarrow}} \mathrm{Bu}_{2} \mathrm{SnO}
$$

$24.8 \mathrm{~g}(0.1 \mathrm{~mole})$ dibutyltin oxide was reacted with $56.8 \mathrm{~g}(0.2 \mathrm{~mole})$ stearic acid $\mathrm{C}_{17} \mathrm{H}_{35} \mathrm{COOH}$ ) by refluxing in toluene for three hours using Dean-Stark apparatus to remove the azeotropic mixture of toluene and water. This is to enable the reaction favour formation of the ester.

$$
\begin{aligned}
& \underset{\text { Stearic acid }}{\stackrel{\mathrm{Bu}_{2} \mathrm{SnO}+2 \mathrm{C}_{17} \mathrm{H}_{35} \mathrm{COOH}}{\text { reflux }}} \\
& \mathrm{Bu}_{2} \mathrm{Sn}\left(\mathrm{OCOC}_{17} \mathrm{H}_{35}\right)_{2}+\mathrm{H}_{2} \mathrm{O} \\
& \text { dibutyltindistearate } \\
& \text { (DBTDST) }
\end{aligned}
$$

The dibutyltin distearate, referred to herein as DBTDST, was characterized by tin analysis and infrared spectroscopy.

\section{Tin analysis}


Tin analysis was carried out using the method reported by Farnsworth and Pekola $^{6}$. 0.2g of DBTDST was weighed separately into three clean and previously weighed sintered crucibles. $1.50 \mathrm{~cm}^{3}$ of concentrated $\mathrm{H}_{2} \mathrm{SO}_{4}(98 \%)$ was added and the mixture heated from the top using bunsen flame until all the $\mathrm{SO}_{2}$ fumes were given off. A dark yellow residue was obtained which was further heated with the flame applied under the crucible placed on a clay triangle on top of a tripod stand. The crucible was burnt thoroughly to remove any carbon deposit. After cooling in a desiccator for $15 \mathrm{hr}$ the crucible $/ \mathrm{SnO}_{2}$ was weighed to obtain the mass of $\mathrm{SnO}_{2}$ by difference. The average of three weights of $\mathrm{SnO}_{2}$ was obtained. The tin content of the DBTDST was calculated from the expression:

$\% \mathrm{Sn}=$ atomic mass of $\mathrm{Sn} \mathrm{X}$ mass of $\mathrm{SnO}_{2} \quad$ x 100 molar mass of $\mathrm{SnO}_{2} \mathrm{X}$ mass of DBTDST(0.2g)

The experimental \%Sn was compared with the theoretical value of $\mathrm{Sn}$ in DBTDST. Further characterization of the tin ester was carried out using infrared spectroscopy as earlier reported ${ }^{7}$.

\section{Incorporation of tin ester into resin and preparation of coatings}

Into a $2000 \mathrm{~cm}^{3}$ quickfit conical flask place $100 \mathrm{~g}$ of PGP resin and $0.399 \mathrm{~g}$

$\left(5 \times 10^{-4}\right.$ moles $)$ of DBTDST. $50 \mathrm{~cm}^{3}$ of thinner (petrol) was then added to the mixture which was thermostated at $35^{\circ} \mathrm{C}$ in a water bath and stirred mechanically for 100 minutes. $10 \mathrm{~cm}^{3}$ of the homogenous liquid was poured onto a glass sheet $(10 \mathrm{~cm} \times 5 \mathrm{~cm})$ and allowed to dry at room temperature. This procedure was repeated twice using $0.798 \mathrm{~g}\left(10 \times 10^{-}\right.$ ${ }^{4}$ mole $)$ and $1.596 \mathrm{~g} \quad\left(20 \times 10^{-4}\right.$ mole $)$ of DBTDST respectively. Thus, for quantitative comparison, the number of mole contained in $100 \mathrm{~g}$ of resin as employed in previous works ${ }^{3,4,8,9,10}$ was used in the present work. These three samples referred to herein as the stabilized samples, therefore, contained 5 , 10 and $20 \times 10^{-4}$ mole \% DBTDST respectively. Using another clean quickfit conical flask, the above procedure was repeated without the tin ester. This served as the control sample.

\section{Outdoor ageing of Coatings}

Glass sheet $(10 \mathrm{~cm} \times 5 \mathrm{~cm} \times 0.2 \mathrm{~cm})$ coated with the control (resin alone) and stabilized samples were mounted on a specially prepared plywood shelve. The plywood shelve was then placed on a roof top so that the glass plates rested at an angle of $45^{\circ}$ to the horizontal.

\section{Determination of hydroxyl (-OH) index}

Films of about $0.02 \mathrm{~cm}$ thickness were removed from the coatings which had set on the glass plates previously exposed on the roof using clean razor blade and placed over the rounded holes( $2 \mathrm{~cm}$ diameter), carved out in paper cards previously. This is to allow ir penetration during the running of the spectra. Thus, spectra of the control and stabilized films (coatings) were obtained at 200 hourly intervals of exposure to outdoor conditions. The $-\mathrm{OH}$ index was obtained using expression similar to one reported earlier. $3,4,9,10$

\section{$-\mathrm{OH}$ index $=$ Absorbance at $3450 \mathrm{~cm}^{-1}$ A $\overline{\text { bsorbance at } 1605 \mathrm{~cm}^{-1}}$}

The $-\mathrm{OH}$ absorbance is at about $3450 \mathrm{~cm}^{-}$ ${ }^{1}$ relative to that of the $\mathrm{C}=\mathrm{C}$ of benzene at $1605 \mathrm{~cm}^{-1}$ The latter absorbance was constant during the period of exposure to outdoor environment and was used as the reference absorbance.

\section{Determination of gel content}

$0.10 \mathrm{~g}$ each of the dry control and stabilized samples was separately weighed into a $20 \mathrm{~cm}^{3}$ volumetric flask. Petrol was added to mark, stoppered and 
left for 72hours in the dark at room temperature with periodic shaking. Thereafter the content was filtered and the residue (gel) was thoroughly washed with petrol and dried to constant weight in the oven at $80^{\circ} \mathrm{C}$.

The gel content $(\%)$ was determined from the expression:

Gel content $(\%)=$

Mass of insoluble fraction $\mathrm{x} 100$ Initial mass of coating $(0.10 \mathrm{~g})$.

The above procedure was repeated at $200 \mathrm{~h}, 400 \mathrm{~h}$ and $600 \mathrm{~h}$ exposure times.

\section{Determination of embrittlement time (ET) of coatings}

The time it takes an exposed film to crack is the embrittlement time, usually determined in hours. The method earlier reported ${ }^{3,4,8,9,10}$ was used. It involved bending the film forward and backward through $180^{\circ}$ at the 200 hourly intervals of exposures until it cracked..

\section{Determination of tensile strength (TS) of coatings}

At 200 hourly intervals of exposure, coatings of $3 \mathrm{~cm} \times 1 \mathrm{~cm}$ and $0.02 \pm$ $0.001 \mathrm{~cm}$ thickness were neatly removed with a clean razor blade and stretched using the INSTRON Tensile Testing machine Model 1026. The gauge length of $1.0 \mathrm{~cm}$ was used. The stress-strain curves at $0 \mathrm{~h}$ and at intervals of exposure to natural weather were plotted from which the tensile strengths(see Figure 1 under 'results and discussion') were obtained as:

Tensile strength $=$ Breaking Load $(\mathrm{N})$ $\left(\mathrm{Nm}^{-2}\right)$

Cross-sectional area $\left(\mathrm{m}^{2}\right)$

\section{RESULTS AND DISCUSSION}

\section{Synthesis of dibutyltin distearate}

Table 1 shows the melting point, result of tin analysis and important i.r. absorptions of DBTDST. The tin analysis gave credence to the successful synthesis of the tin ester as the calculated values compare well with the experimental values.

Table 1: Melting point, tin content and important infrared bands

\begin{tabular}{llll|ccc}
\hline Properties & $\begin{array}{l}\text { M.pt. } \\
\left({ }^{\circ} \mathrm{C}\right)\end{array}$ & $\begin{array}{l}\text { \% Sn } \\
\text { Calc. }\end{array}$ & $\begin{array}{l}\text { \% Sn } \\
\text { Found }\end{array}$ & $\begin{array}{c}\mathrm{U}_{(\text {Sn-Bu rock })} \\
\mathrm{cm}^{-1}\end{array}$ & $\begin{array}{c}\mathrm{U}_{(\mathrm{C}=\mathrm{O} \text { assym })} \\
\mathrm{cm}^{-1}\end{array}$ & $\begin{array}{c}\mathrm{U}_{\text {(Sn-O-C stretch })} \\
\mathrm{cm}^{-1}\end{array}$ \\
\hline DBTDST & $59-61$ & 14.90 & 15.05 & $660(\mathrm{~m})$ & $1550(\mathrm{~s})$ & $985(\mathrm{w})$ \\
\hline
\end{tabular}

$\left(\mathrm{cm}^{-1}, \mathrm{NaCl}\right)$ of DBTDST

The ir results were in agreement with the reported bands of $\mathrm{Sn}-\mathrm{Bu}$ (rock), $\mathrm{C}=\mathrm{O}$ (assym) and Sn-O-C (stretch) absorption bands ${ }^{7}$. These confirm the synthesis of the tin ester.

\section{Oxidation of PGP coatings}

Table 2 shows that the level of formation of hydroxyl compounds is generally higher in the control sample than in the samples containing the 
varying amounts of the DBTDST. The coatings containing 5 and $10 \times 10^{-4}$ mole $\%$ DBTDST have lower $-\mathrm{OH}$ indices, in that order, throughout the period of exposure while the $20 \times 10^{-4}$ mole $\%$ of the additive is generally ineffective in lowering the formation of the $-\mathrm{OH}$ compounds. The sample containing $10 \times 10^{-4}$ mole \% DBTDST with an induction period of $400 \mathrm{~h}$, generally showed the least levels of formation of hydroxyl moiety suggesting that the test tin ester( at $10 \times 10^{-4}$ mole \%) can effectively retard oxidative reactions when incorporated into the PGP coating exposed to weather.

Table 2: Effect of outdoor exposure on the hydroxyl index of PGP alkyd coatings

\begin{tabular}{lcccc}
\hline & \multicolumn{4}{c}{ Hydroxyl Index } \\
\cline { 2 - 5 } Sample & \multicolumn{4}{c}{ Exposure time (h) } \\
\cline { 2 - 5 } & 0 & 200 & 400 & 600 \\
\hline Control (no additive) & 2.52 & 2.40 & 2.00 & 1.94 \\
$5 \times 10^{-4}$ mole\% DBTDST & 1.90 & 1.80 & 1.80 & 1.80 \\
$10 \times 10^{-4}$ mole\% DBTDST & 1.71 & 1.71 & 1.71 & 1.75 \\
$20 \times 10^{-4}$ mole\% DBTDST & 2.00 & 2.30 & 2.00 & 2.10 \\
\hline
\end{tabular}

Formation of $-\mathrm{OH}$ compounds during exposure to outdoor conditions has been attributed to oxidative reactions which are detrimental to the desired properties of polymer films ${ }^{8-10}$. The oxidation is initiated by the formation of free-radicals due to bond-breaking effect of the UV component of the sunlight on the coatings or films ${ }^{2}$.

\section{Gel formation}

Table 3 gives the gel content of the coatings before exposure to weather. The coatings containing the tin esters have higher gel than the control at $0 \mathrm{~h}$ and during outdoor exposure. The coating containing the $10 \times 10^{-4}$ mole $\%$ DBTDST had the greater formation of gel from the onset of exposure.

Table 3: Gel content $(\%)$ of PGP coatings during outdoor exposure

\begin{tabular}{lllll}
\hline & \multicolumn{4}{c}{ Exposure time $(\mathrm{h})$} \\
\cline { 2 - 5 } \multicolumn{1}{c}{ Sample } & 0 & 200 & 400 & 600 \\
\hline Control (no tin ester) & 20 & 60 & 65 & 80 \\
$5 \times 10^{-4}$ mole\% DBTDST & 60 & 60 & 70 & 85 \\
$10 \times 10^{-4}$ mole\% DBTDST & 80 & 90 & 99 & 99 \\
$20 \times 10^{-4}$ mole\% DBTDST & 30 & 70 & 80 & 80 \\
\hline
\end{tabular}

The formation of gel is expected to reduce the level of diffusion/penetration of oxygen into the polymer matrix and so reduce the level of oxidative degradation. The higher gel contents in the coatings containing the test tin ester suggest that the coatings may not be easily oxidized as the control would.

The most probable explanation for these results could be that the thermally sensitive $\mathrm{Sn}-\mathrm{O}$ bond $^{8}$ in the ester 
homolyses yielding, first, the dibutyltin carboxylate radical which can scavenge a polymer radical. The process is repeated until both carboxylate groups in the DBTDST are replaced. Such process will not only reduce the amount of polymer radicals but cross link the polymer chains. The resulting $\mathrm{Sn}-\mathrm{C}$ is most probably less vulnerable to thermal decomposition than the $\mathrm{Sn}-\mathrm{O}$ bond in the tin ester, the Sn - C covalent bond being possibly stronger. The relative strength is based on the fact that the homolytic fission of $\mathrm{Sn}-$ $\mathrm{C}$ or $\mathrm{Sn}-\mathrm{O}$ bond is decided by the resultant electronegativity of $\mathrm{Sn}$ and the moiety of carbon groups attached to it or oxygen and the group attached to it. Therefore, while the electronegativity difference between $\mathrm{Sn}$ and $\mathrm{C}$ or Sn and O may be such that encourages heterolytic fission,the pattern of fission will be the same where the $\mathrm{C}$ and $\mathrm{O}$ are bonded to groups which alter their electronegativities and make them comparable to that of $\mathrm{Sn}$. Consequently, the relative values of thermal parameters are not available to us.

The results of oxidation and gel formation(Tables $2 \& 3$ ) showed that stabilization due to retardative oxidation and increasing gel formation occurred by incorporating 5-10 $\times 10^{-4}$ mole \% DBTDST and increased oxidation at $20 \mathrm{x}$ $10^{-4}$ mole $\%$ of the additive. This may be attributed to excessive dibutyltin radicals obtained at the higher concentration which could abstract $\mathrm{C}-\mathrm{H}$ protons from the polymer (alkyd) chain to produce more of $\mathrm{Sn}-\mathrm{H}$ and oxidation of polymer chain rather than the formation of $\mathrm{Sn}-\mathrm{C}$ in the stabilization reaction

\section{Colour development}

The visual observation of colour employed in previous work is used here ${ }^{10}$. Thus, the colour at $0 \mathrm{~h}$ is the preexposure colour of all samples and the colours at intervals of $200 \mathrm{~h}$ up to $600 \mathrm{~h}$ of exposure to outdoor conditions were observed. Colour change from relatively transparent to yellow films has been attributed to development of conjugated unsaturation $^{11}$. Table 4, therefore, shows that the growth of conjugated unsaturation generally occurred faster in the control than in the coatings containing the test tin ester. It took the control less than $200 \mathrm{~h}$ from the commencement of exposure to change from colourless to light yellow while the samples containing 5,10 and $20 \times 10^{-4}$ mole\% DBTDST had similar colour change after about $400 \mathrm{~h}$, $600 \mathrm{~h}$ and $200 \mathrm{~h}$ respectively. Conjugated unsaturation has been implicated as the precursor to early deterioration of important properties of $\mathrm{PVC}^{11}$ during service life.Thus, the

Table 4: Colour development in PGP coatings during outdoor exposure

\begin{tabular}{lcccc}
\hline & \multicolumn{4}{c}{ Exposure time (h) } \\
\cline { 2 - 5 } & 0 & 200 & 400 & 600 \\
\hline Control (no tin ester) & colourless & light yellow & yellow & Deep yellow \\
Resin $+5 \times 10^{-4}$ mole\% DBTDST & colourless & colourless & colourless & light yellow \\
Resin $+10 \times 10^{-4}$ mole\% DBTDST & colourless & colourless & colourless & colourless \\
Resin $+20 \times 10^{-4}$ mole\% DBTDST & colourless & colourless & light yellow & light yellow \\
\hline
\end{tabular}


presence of the tin esters effectively delayed the formation of conjugated unsaturation thereby stabilizing the PGP alkyd coating against outdoor degradants.

\section{Tensile strength (TS)}

Figure 1 shows the variation in the tensile strength of the control sample and coatings containing 5, 10 and $20 \mathrm{x}$ $10^{-4}$ mole $\%$ DBTDST. It can be seen that while the tensile strength of the control sample decreased sharply with increasing exposure time, the tensile strength of the coatings containing the test tin ester was generally constant. This can be seen from Figure 1 that after the embrittlement of the control sample at about $800 \mathrm{~h}$ outdoor exposure, the coatings containing $10 \times 10^{-}$ 4 mole $\%$ of the test tin ester still had tensile strength values of about $20 \times 10^{6}$ $\mathrm{Nm}^{-2}$. The presence of the tin ester, particularly at $10 \times 10^{-4}$ mole $\%$, could have enhanced the rigidity of the coating probably by increased gel formation as shown in an earlier section.

Mechano-chemical scission has been implicated for the initial degradation of polymers ${ }^{11}$. In Figure 1 the initial TS values (at $0 \mathrm{hr}$ ) showed that the processing had different effects on the unstabilized and stabilized PGP coatings. The TS of the coating containing $10 \times 10^{-4}$ mole $\%$ DBTDST was least deteriorated while that of the unstabilized was most adversely affected. Thereafter, the reduction levels in TS values during exposure were very minimal for samples stabilized with $10 \times 10^{-4}$ mole $\%$ DBTDST. For instance its TS value is 22 $\mathrm{X} 10^{6} \mathrm{Nm}^{-2}$ at $0 \mathrm{~h}$ which reduced to $20 \mathrm{x}$ $10^{6} \mathrm{Nm}^{-2}$ at $800 \mathrm{~h}$ i.e. $9.09 \%$ reduction.

The greatest reduction level in TS value $(77.78 \%)$ occurred in the unstabilized samples within the same period (i.e. $9 \times 10^{6} \mathrm{Nm}^{-2}$ at $0 \mathrm{~h}$ reduced to 2 x $10^{6} \mathrm{Nm}^{-2}$ at $\left.800 \mathrm{~h}\right)$.

\section{Embrittlement time (ET)}

The embrittlement time is the life span index of any coating or film ${ }^{11}$. It can be seen in Table 5 that the coatings containing DBTDST generally had longer ET than the control. The $10 \times 10^{-4}$ mole\% DBTDST was most effective in prolonging the life span of the PGP alkyd coating during outdoor exposure for about 400h (1200h-800h).

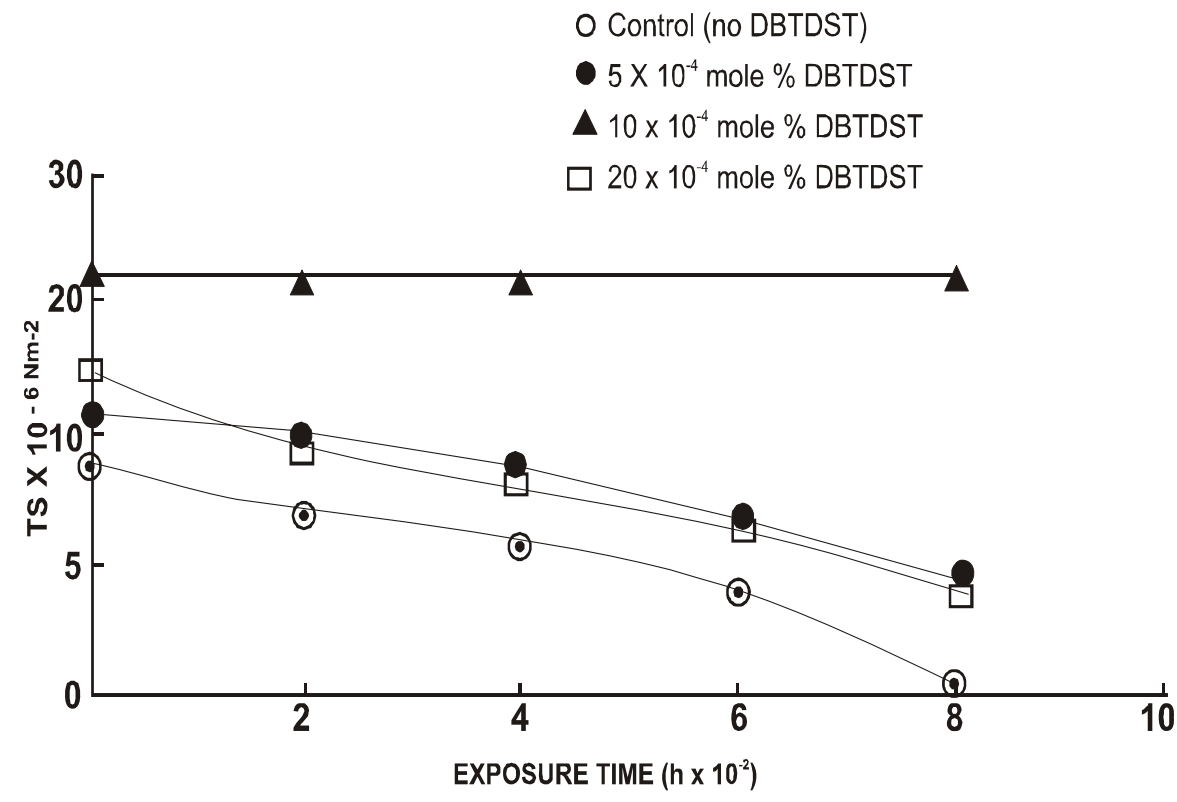


Fig. 1: Weatherability of PGP. Effect of DBTDST on the tensite strength of PGP during outdoor ageing.

Table 5: Embrittlement time (h) (to nearest tens) of PGP alkyd Coatings during outdoor exposure

\begin{tabular}{lc}
\hline Sample & Embrittlement time (h) \\
\hline Control (no additive) & 800 \\
$5 \times 10^{-4}$ mole\% DBTDST & 800 \\
$10 \times 10^{-4}$ mole\% DBTDST & 1200 \\
$20 \times 10^{-4}$ mole\% DBTDST & 1000 \\
\hline
\end{tabular}

The present results show that the degradation and stabilization of the alkyd employed in the present study ( see used in the previous study are quite different from those of the alkyd

Table 6. Hydroxyl index in two Alkyd samples during outdoor Exposure

\begin{tabular}{|c|c|c|c|c|}
\hline \multicolumn{4}{|c|}{ Alkyd (1) } & Alkyd (11) \\
\hline $\begin{array}{l}\text { Exposure } \\
\text { time(h) }\end{array}$ & Control & $\begin{array}{c}\text { Hydroxyl } \\
10 \times 10^{-4} \text { mole } \% \\
\text { DBTDST sample }\end{array}$ & $\begin{array}{l}\text { Index } \\
\text { Control }\end{array}$ & $\begin{array}{l}10 \times 10^{-4} \text { mole } \% \\
\text { DBTDST sample }\end{array}$ \\
\hline 0 & 1.60 & 1.60 & 2.52 & 1.71 \\
\hline 200 & 1.20 & 1.30 & 2.40 & 1.71 \\
\hline 400 & 1.70 & 1.40 & 2.00 & 1.71 \\
\hline 600 & - & - & 1.94 & 2.10 \\
\hline
\end{tabular}

Alkyd I = poly ( pentaerythritol - phthalate )modified with tall oil Alkyd II = poly ( glycerol- phthalate $)$ modified with styrene based on soyabean and dehydrated castor oil.

Table 7 Gel content in two alkyd samples during outdoor explosure.

\begin{tabular}{|c|c|c|c|c|}
\hline & & kyd 1 & \multicolumn{2}{|c|}{ Alkyd $11(\%)$} \\
\hline & & Gel & ontent $(\%$ & \\
\hline \multirow{2}{*}{$\begin{array}{l}\text { Exposure } \\
\text { time(h) }\end{array}$} & & $10 \times 10^{-4}$ mole $\%$ & \multicolumn{2}{|c|}{$10 \times 10^{-4} \mathrm{~mole} \%$} \\
\hline & Control & DBTDST sample & Control & DBTDST sample \\
\hline 0 & 86 & 85 & 20 & 80 \\
\hline 200 & 72 & 86 & 60 & 90 \\
\hline 400 & 62 & 90 & 65 & 99 \\
\hline 600 & - & 89 & 80 & 99 \\
\hline
\end{tabular}

Alkyd 1 - poly ( pentaervthritol - phthalate ) modified with tall oil Alkyd 11 - poly (glycerol -phthalate ) modified with styrene based on sovabean and dehydrated castor oil. 
Moreover, the embrittlement times of the control samples were $500 \mathrm{~h}$ and $800 \mathrm{~h}$ for alkyds I and II respectively. The embrittlement times of the samples stabilized with $10 \times 10^{-4}$ mole $\%$ DBTDST are $1600 \mathrm{~h}$ and $1200 \mathrm{~h}$ for alkyds I and 11 respectively. These differences are consistent with the view that no alkyds have the same degradation and/ or stabilization characteristics ${ }^{2}$. However two other technological parameters i.e. color development and tensile strength, were studied in the present work ( in alkyd 11 ) which were not considered in the

previous article even though the alkyds were stabilized with DBTDST under similar outdoor environment.

\section{CONCLUSION}

The results presented in this article generally point to DBTDST as a possible stabilizer that can redeem PGP based alkyd coating from early deterioration during weathering. The stabilizing activity of the organotin ester can be summarized as follow :

1. The presence of dibutyltin distearate retards formation of oxidation products via a competing reaction between atmospheric oxygen and dibutyltin radicals for polymer chain radicals. Such reaction reduces the amount of macroalkyl radicals that yields oxidized polymer chains.

2. There was generally increased gel in the presence of dibutyltin distearate. This acted, most probably, as a surface covering for reducing further penetration of oxygen into the polymer matrix and hence reduced the level of oxidative degradation of the PGP based alkyd coating.

\section{REFERENCES}

1. Martens C. R. . Waterborne Coatings p.59 Van Nostrand Reinhold Company. New York.1981.

2 Schmid E.V Degradation of Organic coatings by Weathering. p.35 FMJ International Publishers, New York, 1988.

3. Kolawole E.G., Bonire J.J, Peters O., Somorin A.A. and Turoti M. Nig. Jour. of Polym. Sci.\& Techn . 2 (2001)100-112

4. Kolawole, E.G., Bonire, J.J., Peters, O. and Turoti, M. . Nig. Jour.f Polym. Sci. \& Techn., 2 (2001) 132-151.

5. Bentley, $\mathrm{J}$ Organic film formers in Paint and surface coatings,p. 41-60.

Lambourne (ed) John Wiley and Sons, N.Y.1987

6. Farnsworth. M. and Pekola J.. J. Appl. Chem. 31(1954) 40.

7. Bonire, J.J .,Ayoko, G.A., Olurinola, P.F., Ehinmidu J.O.,Jalil N.S,N. and

Omachi, A.A . Metal Based Drugs. 5 (1998) 4233-236.

8. Peters, O.A.,Turoti, M and Adeniyi, J.B. Polym. Deg. \& Stab. 50 (1995) 175182 ,

9. Turoti. M,.,Olayemi J..Y.,Adeniyi, J.B \& Peter, O Polym. Deg. \& Stab. 61(1998) 297-302. .

10. Turoti, M. Adeniyi, J.B, Olayemi, J.Y and Peters O.A. Polm. Deg \& Stab. 63 (1998) 341-347.

11. Cooray, B.B. and Scott, G. Develop. Polym. Stab .2(1980)53-88

accepted 19/10/005

received 20/7/005 